\title{
Optimizing meta-slots for nonblocking elastic optical switching networks
}

\author{
Satoru Ohta * \\ Toyama Prefectural University, 5180 Kurokawa, Imizu-shi, Toyama, 939-0398, Japan.
}

Global Journal of Engineering and Technology Advances, 2021, 07(03), 046-061

Publication history: Received on 29 April 2021; revised on 01 June 2021; accepted on 03 June 2021

Article DOI: https://doi.org/10.30574/gjeta.2021.7.3.0078

\begin{abstract}
Elastic optical network is a promising technology for building flexible and wideband communication systems. This technology features the frequency slot unit (FSU) concept, which defines the bandwidth unit in the frequency domain. The utilization of a specified number of consecutive FSUs allows the bandwidth to be flexibly assigned to a data stream. For a successful elastic optical network operation, the use of nonblocking optical switching networks is indispensable. This paper focuses on two previously proposed W-S-W switching network architectures, namely, WSW1 and WSW2. With regard to WSW1, a previous study elucidated that the hardware complexity, which is evaluated by the number of FSUs, decreases by employing the meta-slot scheme. A meta-slot is a frequency range containing one or more FSUs. Although its effectiveness depends on the sizes of meta-slot classes, the previous study did not present how to optimize the meta-slot class sizes. Moreover, the employment of meta-slots was not considered for WSW2 in the previous study. This paper investigates the optimization of meta-slot class sizes and demonstrates that such optimization is modeled as the shortest path problem. For WSW1, the meta-slot scheme optimized by the shortest path model is compared with the previously reported nonblocking conditions. The result confirms the superiority of the optimized meta-slot scheme. For WSW2, the assignment of meta-slots among S-switches is also essential. The paper models the assignment of metaslots as a bin-packing problem. Thus, the near-optimal assignment can be obtained by a known bin-packing heuristic. For WSW2, the number of S-switches is evaluated for the optimized meta-slot scheme and the previously known nonblocking conditions. The result confirms that the meta-slot scheme is advantageous for WSW2 as well as for WSW1.
\end{abstract}

Keywords: Optical switch; Nonblocking network; Elastic optical network; Shortest path; Bin-packing; Switching network

\section{Introduction}

Elastic optical network [1,2] is a promising technology for building wideband and highly flexible communication networks. This technology enables the coexistence of simultaneous wideband data streams with various bandwidths in a communication network, using the wavelength division multiplexing (WDM) technique. Furthermore, the bandwidth of a data stream can be programmed by choosing an appropriate modulation method.

The elastic optical network technology manages the frequency range of an optical signal modulated by a data stream through frequency slot units (FSUs). An FSU is a constant range that is defined in the frequency domain. The frequency range assigned to a data stream spans an integral number of consecutive FSUs. The data stream bandwidth is determined by the number of FSUs occupied by the modulated optical signal. In [3], the relationship between the FSU concept and modulation methods are explored. In addition, the specification of FSUs is internationally standardized in [4].

\footnotetext{
* Corresponding author: Satoru Ohta

Toyama Prefectural University, 5180 Kurokawa, Imizu-shi, Toyama, 939-0398, Japan. 
A switch is required for each node to build an elastic optical network to adequately route data streams, which utilize different numbers of FSUs. For this purpose, it is important to develop an elastic optical switching network [5-12], which can connect data streams with any number of FSUs without blocking. To this end, a considerable number of studies on the nonblocking conditions of elastic optical switching networks have been conducted. Moreover, a switching network as a topology for data center networks is also vital [13].

A component of elastic optical switching networks is a bandwidth-variable wavelength selective switch (WSS) [1, 2]. It can connect a data stream with any number of FSUs from a single input to any one of multiple outputs. Combining multiple WSSs and couplers makes it possible to construct a space switch (S-switch) and connect data streams between multiple inputs and outputs. However, since an S-switch cannot convert the wavelength, the FSU position used by each connection does not change between its input and output. Therefore, another component required for obtaining full connectivity is a wavelength switch (W-switch), which can convert the wavelength and alter the FSU positions between the input and output.

Previous studies have investigated W-S-W [5-10] or S-W-S [11,12] configurations as elastic optical switching networks. For the W-S-W configuration, two sets of W-switches are placed at the input and output sides, respectively, and linked by $S$-switch(es). This configuration was first introduced by [14] as the $\lambda$-S- $\lambda$ architecture. Meanwhile, W-switches are surrounded by the input/output-side S-switches for the S-W-S configuration, which was explored as the S- $\lambda$-S architecture in [14].

Nonblocking switching networks are categorized into several classes, including strictly nonblocking (SNB), wide-sense nonblocking (WSNB), repackable, and rearrangeable networks [15, 16]. With regard to the W-S-W configuration, previous studies revealed the SNB condition [5, 6], rearrangeable condition [7, 8], and WSNB condition [9, 10]. Among these, the WSNB condition is significant. For this condition, a newly requested connection is never blocked without rerouting any other connections, whereas the hardware amount is smaller than that for the SNB condition.

This paper focuses on the W-S-W configuration and its WSNB condition achieved by the meta-slot scheme proposed by Ohta [10]. A meta-slot is a frequency range containing one or more consecutive FSU(s). For the meta-slot scheme, metaslot classes are defined. Every meta-slot of a class has a constant size, and the number of required FSUs decreases by adequately determining the meta-slot size of each class. By employing meta-slots, [10] demonstrates that the number of FSUs required for a WSW1 inner link significantly decreases compared with the SNB condition, indicating that the required hardware amount is much smaller for the meta-slot scheme.

Although the fundamental advantage of the meta-slot scheme was confirmed in [10], several problems remain to be solved. First, the meta-slot class sizes examined in [10] are not strictly optimal for the number of FSUs. In other words, the number of required FSUs further decreases by optimally deciding the meta-slot class sizes, which may be achieved by exhaustively enumerating every possible combination of meta-slot sizes. However, such exhaustive enumeration is unacceptably time-consuming. Therefore, it is mandatory to establish a fast algorithm to optimize the meta-slot class sizes. Second, the WSW2 architecture was not considered in [10]. Thus, the effectiveness of the scheme is unclear for WSW2. Additionally, the meta-slot design is more complicated for WSW2 because it is necessary to optimize the metaslot class sizes and meta-slot assignment to S-switches. This is a combinatorial optimization problem, which is challenging to obtain the strictly optimal solution.

This paper further investigate the meta-slot scheme to focus on the above problems. The contribution of the paper is summarized as follows.

The optimization of meta-slot class sizes is tackled. It is first shown that the class size determined in [10] is not optimal. Moreover, the paper shows that the class sizes are efficiently optimized by solving the shortest path problem in a network, which models the class sizes and the number of FSUs. The shortest path approach is much faster than exhaustively enumerating possible combinations of meta-slot class sizes. For WSW2, it is crucial to optimally assign meta-slots to multiple S-switches to minimize the number of S-switches. The paper reveals that such optimal assignment of meta-slot classes is a bin-packing problem [17], which is NP-hard. However, practical approximate algorithms are known for this problem. By employing such an algorithm, meta-slots are almost optimally assigned to S-switches.

For WSW1, this paper compares the number of FSUs among the WSNB condition by the meta-slot scheme optimized through the shortest path model, the SNB condition reported in [6], and the conventional WSNB condition by the algorithms reported in [9]. Furthermore, the WSNB condition obtained by the near-optimal assignment of meta-slots through an approximate bin-packing algorithm [17,18] for WSW2 is compared with the SNB condition derived in [6] and the WSNB condition by the algorithms reported in [9]. In both WSW1 and WSW2, the evaluation results confirm 
that the meta-slot scheme is more advantageous than the SNB condition or the WSNB condition obtained by the conventional algorithms.

The remainder of this paper is organized as follows. Section 2 explores the elastic optical network technology, W-S-W optical switching networks, and symbol definitions as preliminaries. In Section3, previous related studies are reviewed. The optimization of the meta-slot class size is presented for WSW1 in Section 4. The section also compares the optimized meta-slot scheme with the previously reported nonblocking conditions. Section 5 examines WSW2. In this section, the optimal assignment of meta-slots among S-switches is investigated, and the meta-slot scheme is compared with the other nonblocking conditions previously known for WSW2. Finally, Section 6 concludes the paper.

\section{Preliminaries}

The elastic optical network technology flexibly provides diverse communication bandwidths using advanced WDM and modulation techniques. A notable feature of this technology is the employment of FSUs. An FSU is a constant frequency range that provides a basis for the spectrum position of a modulated optical signal. Thus, a data stream can utilize an appropriate number of consecutive FSUs to satisfy its bandwidth requirement. Moreover, the number of FSUs occupied by a data stream can be set by selecting an appropriate modulation method [3]. An example of the relationship between FSUs and the spectrum of the optical data stream is presented in Fig. 1. As shown in the figure, the spacing of $12.5 \mathrm{GHz}$ is taken between the center frequencies of two adjacent FSUs [4].

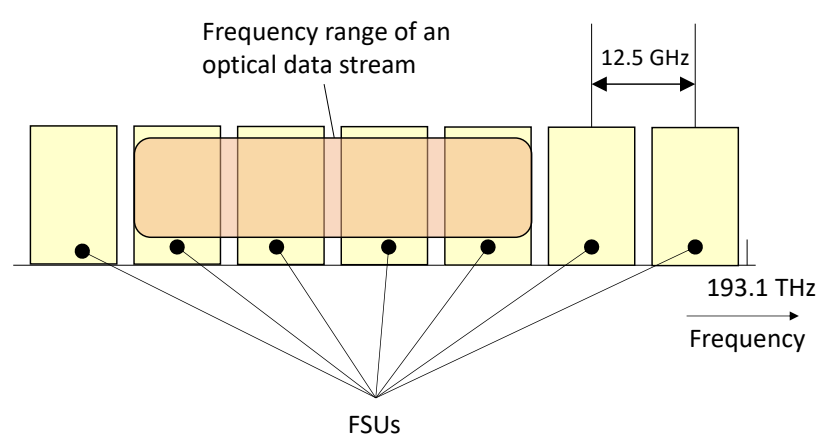

Figure 1 Relationship between FSUs and a frequency range occupied by a data stream in the frequency domain

A tutorial by Jinno [1] explores WSS as a component of elastic optical switching networks, which has one input fiber and multiple output fibers. A WSS can forward a data stream with an arbitrary bandwidth from an input optical fiber to any output optical fiber. A WSS can be implemented with microelectromechanical systems (MEMS) or liquid crystal on silicon (LCoS). Recent advances in the implementations and applications of WSSs are summarized in [19].

Combining multiple WSSs makes it possible to construct a switch with multiple inputs and outputs, as presented in Fig. 2. A data stream arriving from any input optical fibers can be routed to an arbitrary output optical fiber with this switch. However, the FSU position occupied by a connection is fixed between the input and output.

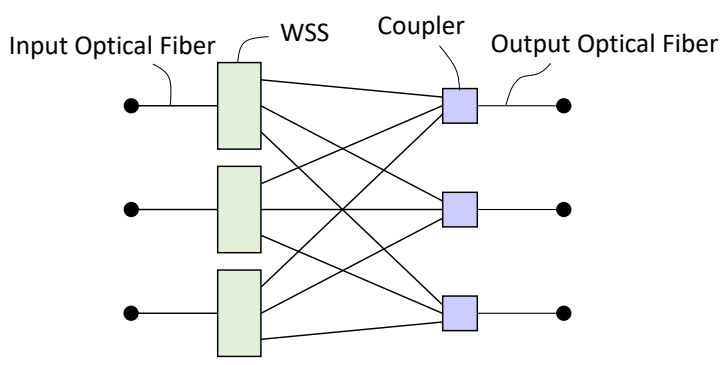

Figure 2 S-switch configured with WSSs and couplers

This type of switch is called bandwidth-variable S-switch in [6]. In this paper, the switch is simply called S-switch. The S-switch can achieve a different permutation between the input and output links for each FSU. 
For achieving full connectivity, it is also imperative to convert the FSU positions assigned to a data stream. This is performed by a tunable waveband converter (TWBC) presented in Fig. 3 (a). A TWBC can be implemented, for example, using the technique explored in $[20,21]$. Furthermore, it is possible to construct a switch with WSSs, TWBCs, and couplers with multiple input/output fibers and exchange the FSU positions between inputs and outputs, as presented in Fig. 3 (b). The configuration shown in Fig. 3 is called W-switches hereafter.

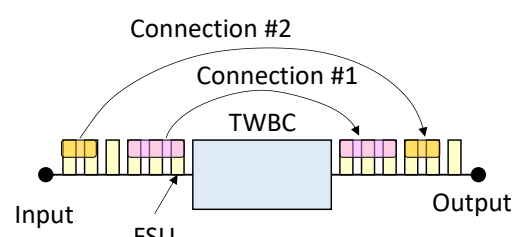

(a)

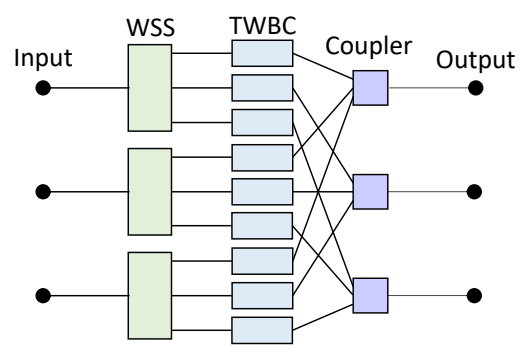

(b)

Figure 3 Two configurations of W-switch, (a) single input/single output configuration, (b) multiple inputs/multiple outputs configuration

The W-S-W (or $\lambda-S-\lambda$ ) architecture is configured by placing two sets of W-switches at the input and output sides, respectively. These input- and output-side $\mathrm{W}$-switches are linked by S-switch(es). In [6], two different W-S-W architectures were examined, presented in Figs. 4 and 5. Fig. 4 depicts the architecture called WSW1, which consists of $r(r>1)$ input-side $\mathrm{W}$-switches, a single S-switch, and $r$ output-side $\mathrm{W}$-switches. Each W-switch has one input and one output. Thus, the S-switch has $r$ inputs and $r$ outputs. Meanwhile, the architecture presented in Fig. 5 is referred to as WSW2, which is constructed by placing $p(p>1) \mathrm{S}$-switches between $r$ input-side and output-side W-switches. To connect a W-switch to every S-switch, each input-side W-switch has $p$ outputs. Similarly, each output-side W-switch has $p$ inputs. For an input-side $\mathrm{W}$-switch, the number of inputs is denoted by $q(q \geq 1)$. An output-side W-switch has the same number of outputs.

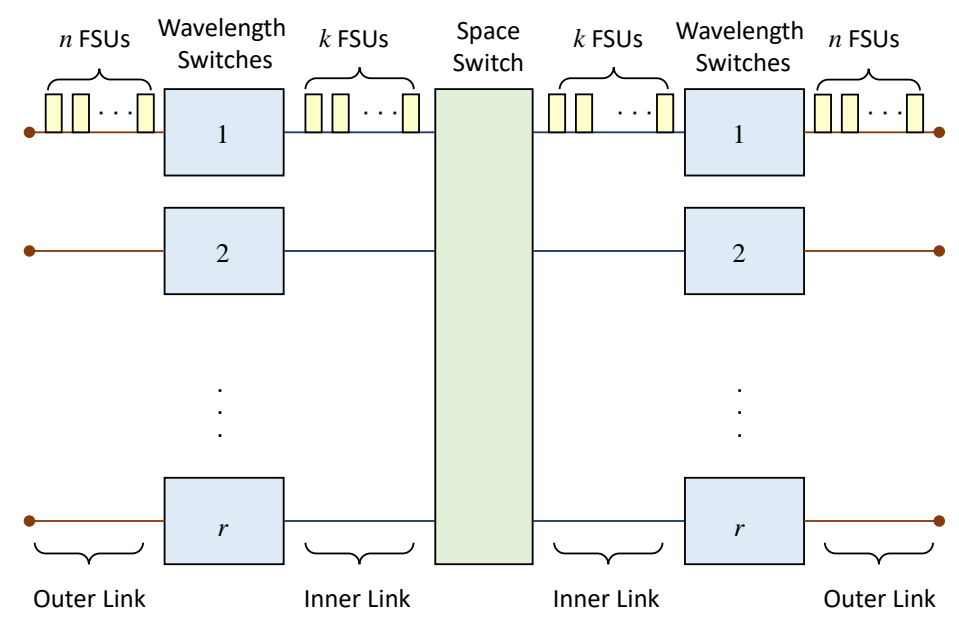

Figure 4 An example of the W-S-W configuration: the WSW1 architecture 


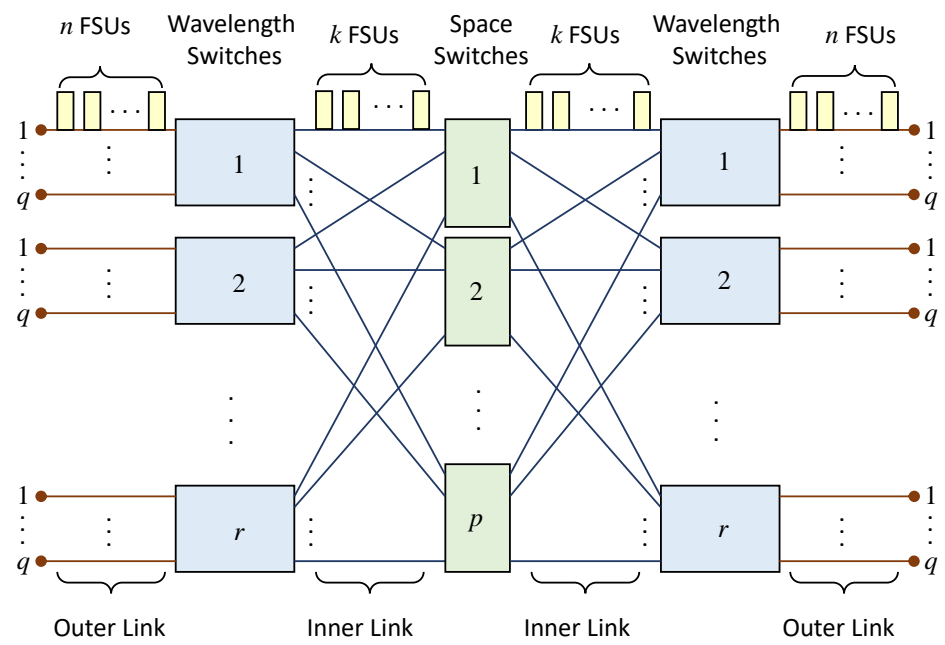

Figure 5 An example of the W-S-W configuration: the WSW2 architecture

This paper uses the terms "inner" and "outer" links. An inner link is defined as an optical fiber connecting a W-switch and an S-switch in WSW1 and WSW2. Conversely, an outer link is an optical fiber attached to an input of an input-side $\mathrm{W}$-switch or an output of an output-side W-switch. Let us assume that $n$ FSUs are provided on each outer link. Let us also assume that $k$ FSUs are available on each inner link. Hereafter, the number of FSUs required for a new connection

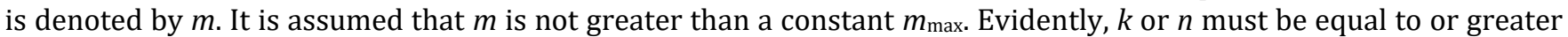
than $m_{\max }$ to establish a connection with the maximum bandwidth.

For WSW1, previous studies [6, 9] have shown the nonblocking conditions as inequalities determining the lower bounds on $k$. The $k$ value should be smaller due to the bandwidth limitation by an optical fiber or the light source. A larger $k$ value is not also desirable due to hardware complexity, such as the number of MEMS mirrors used in WSSs. Thus, it is essential to minimize $k$ for the switching network to be nonblocking. Let $k_{\mathrm{NB}}$ denote this minimum $k$ value.

Nonblocking conditions are expressed differently for WSW2 in previous studies [6, 9]. Namely, the conditions are presented as inequalities on the number of S-switches, $p$, for a fixed value of $k$. It is trivial that the S-switch and W-switch costs are lower for a smaller $p$-value. Thus, it is important to minimize $p$, for which the switching network is nonblocking. Let $p_{\mathrm{NB}}$ denote this minimum $p$-value. The hardware complexity of WSW1 and WSW2 is assessed via $k_{\mathrm{NB}}$ and $p_{\mathrm{NB}}$, respectively.

\section{Related Work}

Studies on nonblocking switching networks have a long history. Its origin is Charles Clos's work [22]. This work revealed the condition, for which a three-stage switching network becomes nonblocking without using any algorithm to decide the routes of connections. This condition is known as SNB. Aside from a class of networks that satisfy the SNB condition, studies identified several other classes of nonblocking switching networks $[15,16]$.

A class of nonblocking switching networks is a rearrangeable network $[23,24]$. For this class, a newly requested connection may be blocked by existing connections. However, such blocking can always be removed if some existing connections are adequately rerouted. A rearrangeable network requires a smaller hardware amount than an SNB network of the same capacity. However, rearrangeable networks have an obvious drawback: data streams may be disturbed by the rearrangements of connections to unblock the system.

Beneš [24] revealed the existence of a switching network, which becomes nonblocking under a particular rule of deciding the route for a newly requested connection. A switching network of this class is WSNB. In [25, 26], the WSNB conditions of a classical Clos network have been comprehensively studied for various routing rules.

With the growing popularity of optical fiber communication, the demand for optical switching networks has also increased. Furthermore, since the WDM technique has become widely used to increase transmission capacity, it becomes necessary to develop optical switches connecting the wavelength paths without blocking. Thus, a significant number of studies on optical switches for WDM systems have been conducted [27-30]. 
As the WDM and modulation techniques have progressed, the concept of an elastic optical network [1] has been proposed. An elastic optical switching network is required to take advantage of this technology, which must connect data streams with different bandwidths without blocking. Although theories have been established for a multi-rate nonblocking switching network [31], an elastic optical switching network must be treated differently from conventional multi-rate switching networks because the FSUs used by a data stream must be consecutively placed in the frequency domain for the elastic optical network technology.

Kabaciński et al. [6] showed the following SNB condition for an elastic WSW1 network:

$$
k_{\mathrm{NB}}=\left\{\begin{array}{cc}
2\left(n-m_{\max }\right) m_{\max }+m_{\max }, & 1 \leq m_{\max } \leq n / 2 \\
\left(n^{2}+n\right) / 2, & n / 2<m_{\max } \leq n
\end{array}\right.
$$

As presented in (1), $k_{\mathrm{NB}}$ is $O\left(n m_{\max }\right)$ or $O\left(n^{2}\right)$ for the SNB condition [10], indicating that the number of FSUs significantly increases for larger values of $n$ or $m_{\max }$. For the WSW2 architecture, the SNB condition is derived by the following value of $p_{\mathrm{NB}}[6]$.

$$
p_{\mathrm{NB}}=\max _{1 \leq m \leq m_{\max }}\left\{\left\lfloor\frac{2(q n-m)}{\left\lfloor\frac{k}{m}\right\rfloor}\right\rfloor+1\right\} .
$$

Kabaciński et al. [6] and Lin [8] reported the rearrangeable conditions for WSW1 and WSW2. However, rearrangeable networks are less practical due to the disturbance of communications during the rearrangements of existing connections, which is avoided for WSNB networks.

The WSNB conditions for WSW1 and WSW2 were also shown by Kabaciński et al. [9]. These conditions were derived by dividing the FSUs on the inner links or S-switches into two or three groups. The groups are separately used, depending on the number of FSUs occupied by a connection. The nonblocking criteria are categorized into the WSNB conditions for these methods since the FSU set, or S-switch is selected for a newly requested connection from the group according to a specific rule.

Among the methods presented in [9], a method developed for WSW1 employs two or three FSU groups. For the case of two groups, one group is used for $m<m_{1}$, whereas the other group is used for the case of $m \geq m_{1}$. Here, $m_{1}$ is a constant, such that $1<m_{1} \leq m_{\max }$ and $m_{1}<n / 2+1$. This method is referred to as the 2 sVarFSU algorithm. Let $k_{1}$ and $k_{2}$ be the number of FSUs assigned to these groups on each inner link. Then, $k_{\mathrm{NB}}$ is derived as follows:

$$
\begin{gathered}
k_{\mathrm{NB}}=k_{1}+k_{2} . \\
k_{1}=2\left(n-m_{1}+1\right)\left(m_{1}-1\right)+m_{1}-1, \\
k_{2}=\max _{m_{1} \leq m \leq m_{\max }} f(m),
\end{gathered}
$$

where

$$
f(m)=2\left\{\left\lfloor\frac{n-m}{m_{1}}\right\rfloor\left(m_{1}+m-1\right)+(n-m) \bmod m_{1}\right\}+m
$$

For WSW2, S-switches are divided into two or three groups. When two groups are used, $p_{1} \mathrm{~W}$-switches are assigned for connections, such that $m<m_{1}$. Meanwhile, $p_{2} \mathrm{~W}$-switches are assigned to connections, such that $m_{1} \leq m \leq m_{\text {max }}$. This method is called the 2sVarSWITCH algorithm. For this operation, $p_{\mathrm{NB}}$ is determined as follows:

$$
\begin{gathered}
p_{\mathrm{NB}}=p_{1}+p_{2}, \\
p_{1}=\max _{1 \leq m<m_{1}}\left\{\left\lfloor\frac{2(q n-m)}{\left\lfloor\frac{k}{m}\right\rfloor}\right\rfloor+1\right\}, \\
\left.p_{2}=\max _{m_{1} \leq m \leq m_{\max }}\left\{\mid \frac{2(q n-m)}{m_{1}\left|\frac{k}{m_{1}+m-1}\right|+a}\right\rfloor+1\right\},
\end{gathered}
$$

where $a$ takes one of $0, m_{1}$, and $k \bmod \left(m_{1}+m-1\right)-m+1$, depending on the values of $k, m_{1}$, and $m$. In [9], the methods for dividing FSUs or S-switches into three groups are also presented. These methods are called 3sVarFSU and 3sVarSWITCH algorithms for WSW1 and WSW2, respectively. It was found that the value of $k_{\mathrm{NB}}$ or $p_{\mathrm{NB}}$ is smaller for the WSNB conditions obtained using these algorithms than that for the SNB conditions. However, it is unclear whether the employment of two or three FSU (or S-switch) groups is sufficient to minimize the hardware amount. Moreover, it is unknown how to efficiently optimize the parameters used in the algorithms, such as $m_{1}$ for a given condition. 


\subsection{Meta-Slot Scheme}

Ohta [10] presented another nonblocking condition for WSW1. This nonblocking condition is derived by employing the meta-slot concept. When meta-slots are used, $k_{\mathrm{NB}}$ is $O\left(n \log m_{\max }\right)$, whereas $k_{\mathrm{NB}}$ is as large as $O\left(n m_{\max }\right)$ for the SNB condition. Thus, the method is more advantageous for larger $m_{\max }$. Since the method decides the FSUs used for a connection according to the meta-slot positions, the nonblocking condition is a WSNB condition.

A meta-slot is a frequency range covering an integral number of consecutive FSUs in the frequency domain. Thus, a meta-slot can be considered as a larger slot, which can contain multiple FSUs. Furthermore, a meta-slot is a logical concept used to determine the FSUs served for a new connection on inner links. In other words, meta-slots are maintained only in the control software that hunts the FSUs for a new connection. Thus, the use of meta-slots does not require any hardware modifications.

For WSW1 and WSW2, meta-slots with different sizes coexist on each inner link. Then, a meta-slot with an appropriate size is selected for setting up a newly requested connection. Specifically, $u(u \geq 1)$ classes of meta-slots, $M_{1}, M_{2}, M_{3}, \ldots$, $M_{u}$, are defined. A meta-slot of class $M_{v}(1 \leq v \leq u)$ is composed of $s_{v}$ consecutive FSUs. The constant $s_{v}$ is referred to as the size of a class $M_{v}$ meta-slot. Without loss of generality, $s_{v}$ is assumed to satisfy the following relation:

$$
s_{v}<s_{v+1}, 1 \leq v<u
$$

Additionally, let us define $s_{0}=0$ for simplicity of description, although class $M_{0}$ does not exist. The meta-slots of each class must be placed at the same position in the frequency domain for every inner link in WSW1, which is trivially achievable. First, the size of each meta-slot class is determined, and the number of meta-slots is also obtained for the class. Then, the meta-slots of each class are placed in the order of $M_{1}, M_{2}, M_{3}, \ldots, M_{u}$ from the lower (or higher) frequency for every inner link. This class placement enables the S-switch to always connect an idle meta-slot on each input link to an idle meta-slot of the same class and position on an arbitrary output link.

For the meta-slot scheme, a newly requested connection is set up as follows. The connection is established through a meta-slot of class $v$ that satisfies the following:

$$
s_{v-1}<m \leq s_{v} .
$$

Namely, the connection uses the meta-slot with the smallest size but not smaller than the number of required FSUs. Moreover, a meta-slot is exclusively used by a single connection; a meta-slot partly used by an existing connection is not used for any other connections, even if it contains idle FSUs. The relationship among meta-slots, FSUs, and the connection spectrum is presented in Fig. 6.

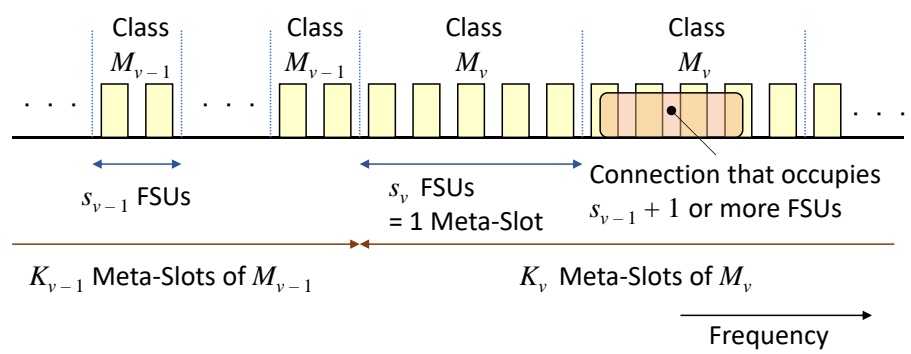

Figure 6 Relationship among meta-slots, FSUs, and the frequency range occupied by a connection

The meta-slot scheme has three advantages. First, the scheme prevents a narrowband connection from blocking a newly requested wideband connection. This advantage is obtained as the narrowband and wideband connections use different meta-slot classes and do not conflict. Second, all the connections set up through class $M_{v}$ meta-slots are considered to occupy the same number of FSUs. In other words, the number of FSUs is rounded up from $m$ to $s_{v}$. As a result, the nonblocking condition can be analyzed as the single rate model for each meta-slot class, and thus, the analysis is greatly simplified. Finally, the control complexity decreases to hunt idle consecutive FSUs for a newly requested connection.

The WSNB condition is described as follows [10]. Let $K_{v}$ denote the number of class $M_{v}$ meta-slots required for the system to be nonblocking. Then, WSW1 managed by the meta-slot scheme is considered nonblocking if

$$
K_{v}=2\left\lfloor\frac{n}{s_{v-1}+1}\right\rfloor-1,1 \leq v \leq u .
$$


$k_{\mathrm{NB}}$ is immediately obtained as follows with the use of $K_{v}$

$$
k_{\mathrm{NB}}=\sum_{v=1}^{u} K_{v} s_{v}
$$

In [10], a comparison between the WSNB condition and the SNB condition is presented, as well as the superiority of the meta-slot scheme. However, the WSNB condition by the meta-slot scheme is not compared with the WSNB conditions derived for the algorithms reported in [9]. It is also unclear whether the sizes of meta-slot classes explored in [10] are optimal or not. Additionally, for WSW2, the design and effectiveness of the meta-slot scheme are not investigated in [10].

\section{Meta-Slot Optimization for WSW1}

\subsection{Optimization of Meta-Slot Class Sizes}

For the meta-slot scheme, the value of $k_{\mathrm{NB}}$ depends on parameters $u$ and $s_{v}$, which must be optimally determined to minimize $k_{\mathrm{NB}}$. Reference [10] shows that $k_{\mathrm{NB}}$ is $O\left(n \log m_{\max }\right)$ if the meta-slot scheme is applied to WSW1. This result is obtained by setting,

$$
\begin{gathered}
u \triangleq\left\lceil\log _{d} m_{\text {max }}\right\rceil, \\
s_{v} \triangleq\left\{\begin{array}{cc}
0, & v=0 \\
\left\lfloor d^{v}\right\rfloor, & 0<v<u, \\
m_{\text {max }}, & v=u
\end{array}\right.
\end{gathered}
$$

where $d$ is a constant $(d>1)$. However, the values of $u$ and $s_{v}$ of (14) and (15) are not necessarily optimal to minimize $k_{\mathrm{NB}}$. This characteristic is evident from the following theorem.

Theorem 1 For WSW1 operated by the meta-slot scheme, if $k_{\mathrm{NB}}$ is minimized,

$$
s_{1}=1 \text {. }
$$

Proof: Suppose that we have meta-slot classes $M_{1}, M_{2}, \ldots, M_{u}$, for which $s_{1} \geq 2$. Let $k_{0}$ denote the value of $k_{\mathrm{NB}}$ for this metaslot class set. Then, consider another set of meta-slot classes $\widehat{M}_{1}, \widehat{M}_{2}, \ldots, \widehat{M}_{u+1}$. Let the sizes of these meta-slot classes be $\hat{s}_{1}, \hat{s}_{2}, \ldots, \hat{s}_{u+1}$, respectively.

Here, $\hat{s}_{v},(1 \leq v \leq u+1)$ is defined as follows:

$$
\begin{gathered}
\hat{s}_{1}=1, \\
\hat{s}_{v}=s_{v-1}, 2 \leq v \leq u+1 .
\end{gathered}
$$

Let $k_{1}$ denote the value of $k_{\mathrm{NB}}$ when $\widehat{M}_{1}, \widehat{M}_{2}, \ldots, \widehat{M}_{u+1}$ are used. Then, from (12) and (18), the number of FSUs required for class $M_{2}, M_{3}, \ldots, M_{u}$ meta-slots equals that for class $\widehat{M}_{3}, \widehat{M}_{4}, \ldots, \widehat{M}_{u+1}$ meta-slots. Thus, the difference between $k_{0}$ and $k_{1}$ is determined by the number of FSUs required for $M_{1}, \widehat{M}_{1}$, and $\widehat{M}_{2}$. From (12) and the definition of $s_{0}$, the number of $M_{1}$ meta-slots, $K_{1}$, is

$$
K_{1}=2 n-1 \text {. }
$$

Let the numbers of $\widehat{M}_{1}$ and $\widehat{M}_{2}$ meta-slots be $\widehat{K}_{1}$. and $\widehat{K}_{2}$, respectively. From (12), these values are

$$
\begin{gathered}
\widehat{K}_{1}=2 n-1, \\
\widehat{K}_{2}=2\left\lfloor\frac{n}{2}\right\rfloor-1 \leq n-1 .
\end{gathered}
$$

The difference between $k_{1}$ and $k_{0}$ is

$$
k_{0}-k_{1}=K_{1} s_{1}-\widehat{K}_{1} \hat{s}_{1}-\widehat{K}_{2} \hat{s}_{2}
$$

From (19), (20), (21) and (22),

$$
k_{0}-k_{1} \geq n s_{1}-2 n+1 \text {. }
$$

Since $s_{1} \geq 2$, 


$$
k_{0}-k_{1} \geq 1 \text {. }
$$

Thus, the meta-slot classes with $s_{1}=1$ yield a smaller value of $k_{\mathrm{NB}}$ than those with $s_{1} \geq 2$.

Since the $s_{1}$ value given by (15) is not necessarily 1 , the $s_{v}$ values of (15) do not yield the optimal meta-slot classes, which minimize $k_{\mathrm{NB}}$. Meanwhile, (15) suggests that $s_{1}$ equals 1 if $d<2$. However, the numerical computation shows that $k_{\mathrm{NB}}$ is not minimized for $d<2[10]$.

Essentially, the set of meta-slot classes can be optimized through an exhaustive search of every possible combination of $u, s_{1}, s_{2}, \ldots, s_{u}$. Nevertheless, the exhaustive search is impractically time-consuming. Fortunately, this optimization is efficiently performed in a polynomial time through the employment of a network model.

As observed from (12), $K_{v}$ is determined by a given constant $n$ and a variable $S_{v-1}$. The number of the FSUs required for class $M_{v}$ meta-slots is $s_{v} K_{v}$. Thus, the number of FSUs is determined by two variables, $s_{v-1}$ and $s_{v}$, for class $M_{v}$ using (12).

A network is a powerful approach for representing the relationship between two things. For this case, let two vertices represent $s_{v-1}$ and $s_{v}$. Then, let us define a directed edge $\left(s_{v-1}, s_{v}\right)$. Representing the number of FSUs required for $M_{v}$ metaslots as the edge length is possible for this edge. Based on this concept, a network is constructed by defining the vertices as all possible values of $s_{v}^{\prime}$ 's and setting the edges between the vertices. Specifically, vertices set $V$ is defined as follows:

$$
V=\left\{0,1,2, \ldots, m_{\max }\right\} .
$$

Edge set $E$ is

$$
E=\{(i, j) \mid i, j \in V, i<j\}
$$

Let $l_{i, j}$ denote the length of $(i, j)$. Edge length $l_{i, j}$ is the number of FSUs required for the meta-slot class $M_{v}$ with assuming $s_{v}=j$ and $s_{v-1}=i$. Namely, by multiplying $j$ and $K_{v}$,

$$
l_{i, j}=\left(2\left\lfloor\frac{n}{i+1}\right\rfloor-1\right) j .
$$

Based on the above definitions, a directed network $G(V, E)$ is constructed. In Fig. 7, an example of $G(V, E)$ is presented, which displays the case of $m_{\max }=5$.

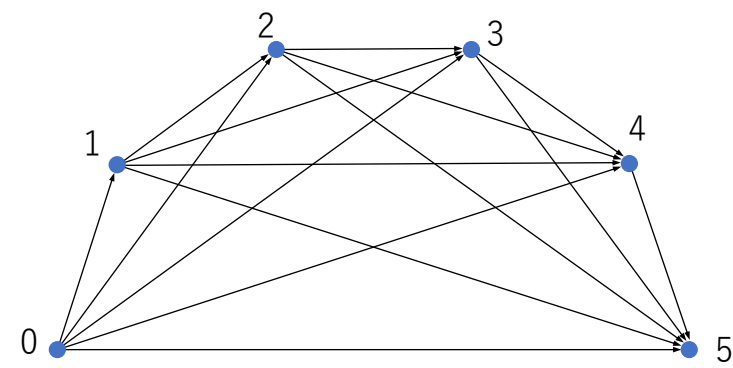

Figure 7 Network model to optimize meta-slot class sizes $s_{v}{ }^{\prime}$ s for $m_{\max }=5$

Theorem 2 In $G(V, E)$, the vertices in the shortest path from 0 to $m_{\max }$ represent the optimal values of $s_{1}, s_{2}, \ldots, s_{u}$.

Proof: In $G(V, E)$, we focus on a directed path from vertex 0 to vertex $m_{\max }$. Let the $v_{0}(=0), v_{1}, v_{2}, \ldots, v_{u}\left(=m_{\max }\right)$ denote the vertices on this path. Then, it is possible to associate the meta-slot sizes with this path by setting $s_{1}=v_{1}, s_{2}=v_{2}, \ldots, S_{u}$ $=v_{u}$. For this setting, the number of FSUs for class $M_{i}$ is expressed by the length of $\left(v_{i-1}, v_{i}\right)$. Furthermore, the sum of the edge lengths on the path equals the total number of required FSUs, $k_{\mathrm{NB}}$. Also, every possible combination of $u, s_{1}, s_{2}, \ldots$, $S u$ is associated with a certain path from 0 to $m_{\max }$. Thus, the minimum value of $k_{\mathrm{NB}}$ is obtained as the distance of the shortest path from 0 to $m_{\max }$ and the optimal meta-slot sizes are given as the vertices on the shortest path.

By Theorem 2, the optimal set of $s_{1}, s_{2}, \ldots, s_{u}$ is easily obtained by running the shortest path algorithm [32] with vertex 0 set as the origin. Moreover, since $G(V, E)$ is constructed by $m_{\max }+1$ vertices, it is evidently possible to construct the 
graph and execute the shortest path algorithm in a polynomial time for the problem size $m_{\max }$. Thus, the optimal metaslot sizes and the minimum $k_{\mathrm{NB}}$ are obtained in a reasonable computational time.

\subsection{Comparison with Previous Results}

First, let us assess the effectiveness of the meta-slot class optimization using the shortest path algorithm. For this purpose, $k_{\mathrm{NB}}$ was evaluated for the optimized meta-slot class and the method explored in [10]. For the latter case, $u$ and $S_{v}$ 's were determined by (14) and (15). For (15), the value of constant $d$ was 2.7183. This value of $d$ is expected to yield a good solution [10]. The result is presented in Fig. 8. In the figure, the $\mathrm{x}$-axis is $m_{\max }$, whereas the y-axis is $k_{\mathrm{NB}}$. The number of FSUs on each outer link, $n$, was set to 120 . Since the number of input/output-side $\mathrm{W}$-switches, $r$, does not affect $k_{\mathrm{NB}}$ for any methods, $r$ was not considered. The figure shows that $k_{\mathrm{NB}}$ visibly decreases by the shortest path optimization compared to the method of [10].

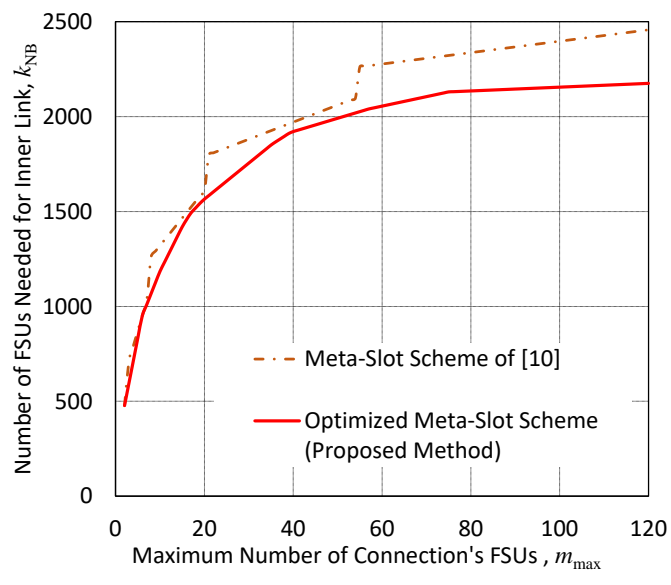

Figure 8 Relationship between $m_{\max }$ and $k_{\mathrm{NB}}$ for the meta-slot scheme optimized by the shortest path algorithm and the meta-slot scheme by the parameters determined by the method presented in [10]

Next, the optimized meta-slot scheme was compared with the SNB condition [6] and the WSNB conditions derived for the algorithms reported in [9]. Fig. 9 plots $k_{\mathrm{NB}}$ against $m_{\max }$ for these cases. In the figure, $n$ was set to 120. For the WSNB conditions by the algorithms of [9], parameters $m_{1}$ (for 2sVarFSU and 3sVarFSU) and $m_{2}$ (for 3sVarFSU) are selected to minimize $k_{\mathrm{NB}}$.

The advantage of the proposed meta-slot scheme can be clearly seen in Fig. 9. The figure shows that the $k_{\mathrm{NB}}$ value is much smaller for the 2sVarFSU algorithm than for the SNB condition. Furthermore, the value further decreases by employing the 3sVarFSU algorithm for $m>20$. However, the optimized meta-slot scheme yields smaller $k_{\mathrm{NB}}$ values than both of the 2sVarFSU and 3sVarFSU algorithms.

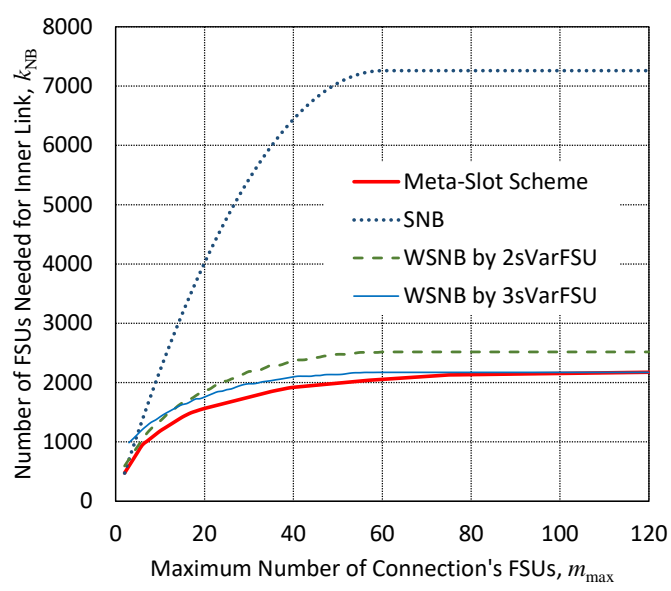

Figure 9 Comparison between the optimized meta-slot scheme and previous nonblocking conditions for WSW1 through the number of FSUs required on an inner link versus the maximum number of FSUs occupied by a connection 
As can be seen from Fig. 9, the $k_{\mathrm{NB}}$ value does not significantly differ between the meta-slot scheme and the WSNB condition by $3 \mathrm{sVarFSU}$, particularly when $m_{\max }>80$. However, the advantage of the meta-slot scheme increases for larger $n$ values. This characteristic is presented in Fig. 10, which compares the optimized meta-slot scheme and the WSNB conditions by the $2 \mathrm{sVarFSU} / 3 \mathrm{sVarFSU}$ algorithms. In the figure, the $\mathrm{x}$-axis denotes $n$, whereas the $y$-axis denotes $k_{\mathrm{NB}}$. The value of $m_{\max }$ is set equal to $n$. For the conventional WSNB conditions by the $2 \mathrm{sVarFSU}$ and $3 \mathrm{sVarFSU}$ algorithms, $m_{1}$ and $m_{2}$ are optimized to minimize $k_{\mathrm{NB}}$. As shown in Fig. 10, the difference between the methods becomes greater when $n$ is larger. When $n=m_{\max }=1000, k_{\mathrm{NB}}$ for the meta-slot scheme is smaller than half of that for $2 \mathrm{sVarFSU}$ and $77 \%$ of 3sVarFSU.

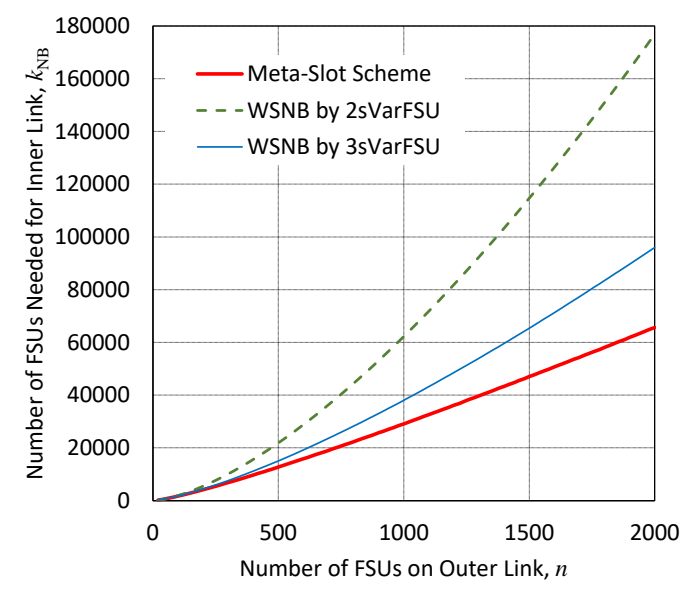

Figure 10 Comparison between the meta-slot scheme and previous WSNB conditions for WSW1 through the number of FSUs required on an inner link versus the number of FSUs on an outer link when $m_{\max }=n$

Fig. 11 presents the characteristics of the meta-slot scheme and other conventional WSNB conditions when $m_{\max }$ is fixed to a constant, i.e., 100 . This figure also demonstrates that when $n$ is large, the meta-slot scheme is more advantageous than the 2sVarFSU or 3sVarFSU algorithms. Based on Figs. 10 and 11, it is concluded that the WSNB condition by the optimized meta-slot scheme decreases the number of FSUs for inner links compared to those by the previously reported algorithms.

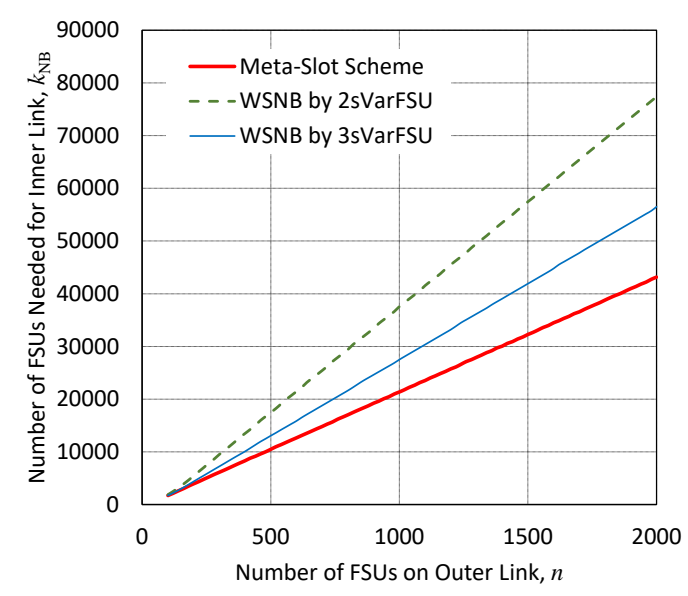

Figure 11 Comparison between the meta-slot scheme and previous WSNB conditions for WSW1 through the number of FSUs required on an inner link versus the number of FSUs on an outer link when $m_{\max }=100$ 


\section{Meta-Slot Optimization for WSW2}

\subsection{Nonblocking Condition}

The meta-slot scheme is also applicable to the WSW2 architecture. The scheme employs $u$ classes $M_{1}, M_{2}, M_{3}, \ldots, M_{u}$, each of which contains $s_{v}$ FSUs similarly as for the case of WSW1. For a connection occupying $m$ FSUs, the class $M_{v}$ is selected, such that $s_{v}$ is the smallest and not smaller than $m$.

The position of each meta-slot class must be the same for every inner link to enable the full connection of meta-slots in an S-switch. In addition, the positions of the meta-slot class may differ between S-switches, which is achievable as follows. First, it is decided how many meta-slots of classes $M_{1}, M_{2}, M_{3}, \ldots, M_{u}$ are assigned to each of the $p$ S-switches. Then, for each S-switch, the meta-slots of a class are placed in the order of $M_{1}, M_{2}, M_{3}, \ldots, M_{u}$ from the lower (or higher) frequency for every inner link. This setting makes it possible for an S-switch to connect every meta-slot from an inputside W-switch to an output-side W-switch. This configuration is presented in Fig. 12. To know whether such configuration is correct, let $1,2, \ldots, K_{v}$ denote class $M_{v}$ meta-slots on $p$ outputs of a certain input-side W-switch. It is also assumed that FSUs are indexed $1,2, \ldots, k$. Then, if meta-slot $t\left(1 \leq t \leq K_{v}\right)$ is located at an S-switch $X$ and an FSU range $[Y, Z](1 \leq Y<Z \leq k)$ for this W-switch, $t$ is apparently assigned to the same pair of S-switch $X$ and FSU range [Y, $Z$ ] for any output-side $\mathrm{W}$-switches. Thus, $t$ can be connected from an input-side $\mathrm{W}$-switch to any one of output-side Wswitches, in which $t$ is idle.

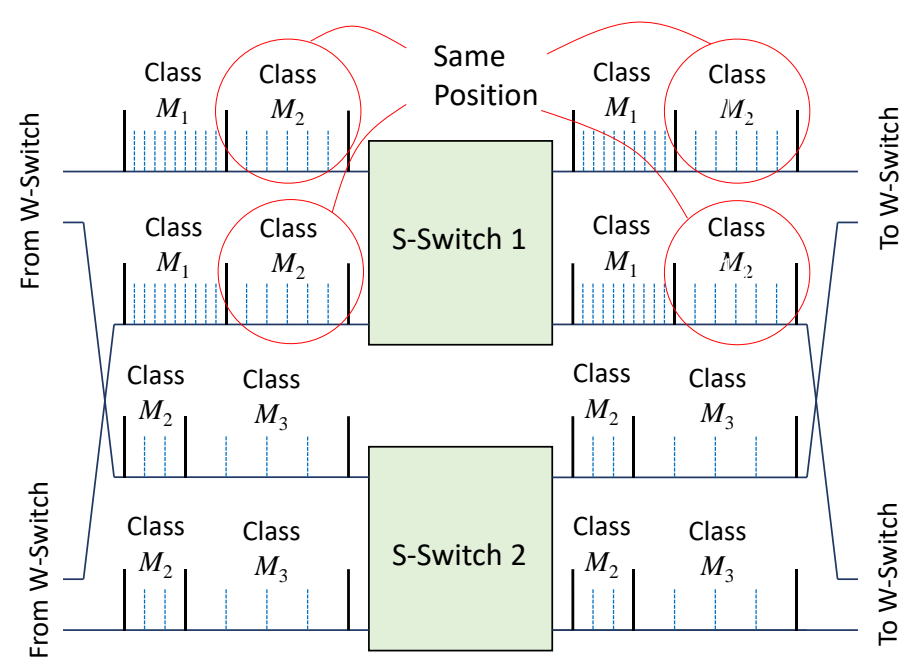

Figure 12 An example of meta-slot alignment among S-switches for the WSW2 architecture.

For WSW2 to be nonblocking, the total number of meta-slots should be determined using the following theorem.

Theorem 3 Let $K_{v}$ be the total number of class $M_{v}$ meta-slots provided on $p$ inner links attached to each W-switch in WSW2. Then, WSW2 is nonblocking if

$$
K_{v}=2\left\lfloor\frac{n q}{s_{v-1}+1}\right\rfloor-1
$$

Proof: Assume that a new connection is required from an input-side W-switch $I$ to an output-side $\mathrm{W}$-switch $O$. There are $n q$ FSUs on $q$ outer links of $I$ (or $O$ ). Since a new connection is requested, there are at least $m$ consecutive idle FSUs on the outer links of $I$ ( or $O$ ). Thus, the existing connections set up through $I$ (or $O$ ) consume at most $n q-m$ FSUs. Let $x$ and $y$ denote the number of class $M_{v}$ meta-slots used by the existing connections passing through $I$ and $O$, respectively. Then, since an $M_{v}$ meta-slot class is used by the connections consuming $s_{v-1}+1$ or more FSUs,

$$
x \leq\left\lfloor\frac{n q-m}{s_{v-1}+1}\right\rfloor .
$$

From (11),

$$
x \leq\left\lfloor\frac{n q-\left(s_{v-1}+1\right)}{s_{v-1}+1}\right\rfloor=\left\lfloor\frac{n q}{s_{v-1}+1}\right\rfloor-1 .
$$


Similarly,

$$
y \leq\left\lfloor\frac{n q}{s_{v-1}+1}\right\rfloor-1
$$

If $x+y+1$ or more meta-slots of class $M_{v}$ are provided on the inner links between $I$ and $O$, at least one idle meta-slot exists. The connection can be set up between this idle meta-slot. Thus, this proves the theorem.

\subsection{Minimizing the Number of S-Switches}

Intuitively, $p_{\mathrm{NB}}$ is smaller if the total number of FSUs required for meta-slots is minimal. Therefore, the total number of FSUs is minimized by optimizing meta-slot class size $s_{v}$, which is achieved by the shortest path algorithm described for WSW1 in Section 4.1. For WSW2, the same approach is usable, except for the replacement of $n$ with $n q$ in (27), which determines the edge length and can be easily understood by comparing (12) with (28).

For given constants $n, q, k$, and meta-slot class sizes, $p_{\mathrm{NB}}$ is determined by optimally distributing $K_{v}$ meta-slots of class $M_{v}$ among $p$ S-switches. This is a bin-packing optimization problem [17]. Namely, the total number of items is $\sum_{v} K_{v}$. Among these, there are $K_{v}$ items of size $s_{v} / k$. The problem is packing these items to the minimum number of unitcapacity bins. Thus, after determining $s_{v}$ and $K_{v}$ to minimize the total number of FSUs, meta-slots should be optimally assigned to $\mathrm{S}$-switches as a bin-packing problem.

It is known that the bin-packing problem is NP-hard. However, several powerful approximation algorithms exist for the problem $[17,18]$. The use of such an algorithm makes it possible to calculate $p_{\mathrm{NB}}$ and the assignment of meta-slots to Sswitches within a reasonable computational time. It is also expected that the obtained solution is very close to the strict optimum.

\subsection{Comparison with Previous Results}

The meta-slot scheme for WSW2 was compared with the conventional SNB [6] and WSNB conditions [9] through numerical evaluation. The methods were compared in terms of the number of required S-switches, $p_{\mathrm{NB}}$, where parameters $n, k, q$, and $m_{\max }$ were given. The number of input/output-side $\mathrm{W}$-switches, $r$, does not affect $p_{\mathrm{NB}}$ in any methods and thus was not considered.

For the meta-slot scheme, the meta-slot class size $s_{v}$ was optimized by the shortest path algorithm. Then, the meta-slots were assigned to S-switches by the First-Fit Decreasing algorithm [17, 18], which approximately solves a bin-packing problem. As the number of bins, the value of $p_{\mathrm{NB}}$ is obtained.

For the conventional WSNB conditions, the 2sVarSWITCH and 3sVarSWITCH algorithms were considered. Both algorithms use parameter $m_{1}$, with the latter algorithm also employing parameter $m_{2}$. These parameters were set to minimize $p_{\mathrm{NB}}$.

Fig. 13 presents $p_{\mathrm{NB}}$ versus $m_{\max }$ with constant $n, k$, and $q$. In this figure, $n, k$, and $q$ are set to 100,100 , and 4 , respectively. As shown in the figure, the difference between the methods becomes more significant as $m_{\max }$ increases. The value of $p_{\mathrm{NB}}$ is much smaller for the WSNB conditions than for the SNB condition. Among the conventional WSNB conditions, the 3 sVarSWITCH algorithm yields smaller $p_{\mathrm{NB}}$ values than the 2sVarSWITCH algorithm. However, the meta-slot scheme, optimized through the shortest path and bin-packing algorithm, performs better than the 3sVarSWITCH algorithm. 


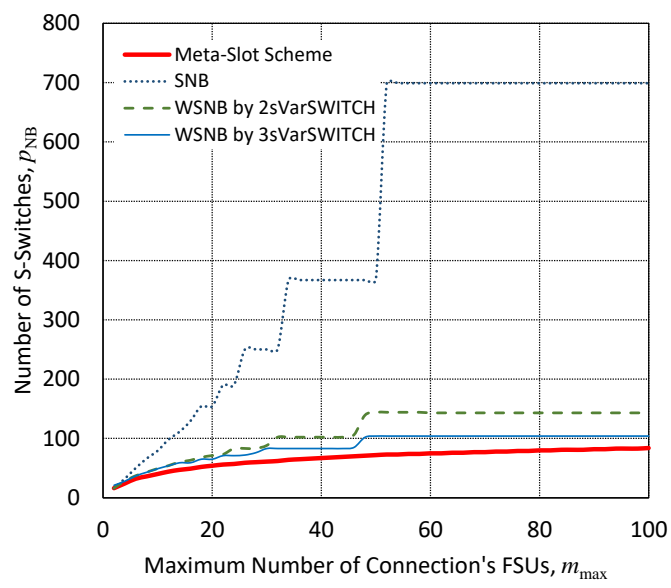

Figure 13 Comparison between the meta-slot scheme and previous nonblocking conditions for WSW2 through the number of required space switches versus the maximum number of FSUs occupied by a connection when $n=k=100$

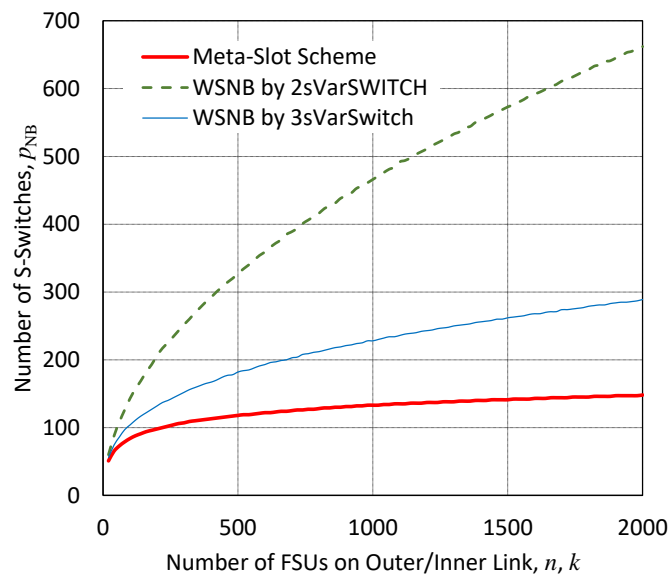

Figure 14 Comparison between the meta-slot scheme and previous nonblocking conditions for WSW2 through the number of required space switches versus the number of FSUs on an outer/inner link when $n=k=m_{\max }$

Fig. 14 plots $p_{\text {NB }}$ against $n$. This figure compares three WSNB conditions: the meta-slot scheme, 2sVarSWITCH algorithm, and 3sVarSWITCH algorithm. Parameters $k$ and $m_{\max }$ were set equal to $n$, whereas parameter $q$ was set to 4 . The figure shows that $p_{\mathrm{NB}}$ is larger for $2 \mathrm{sVarSWITCH}$ than for the other two schemes. In addition, the meta-slot scheme outperforms 3 sVarSWITCH. The advantage of the meta-slot scheme becomes greater for larger $n$ values, as shown in the figure. For example, when $n=100$, the $p_{\mathrm{NB}}$ value for the meta-slot scheme is $80.8 \%$ of that for $3 \mathrm{sVarSWITCH}$. Meanwhile, if $n$ is 2000 , the $p_{\mathrm{NB}}$ value for the meta-slot scheme decreases to $51.2 \%$ of that for $3 \mathrm{sVarSWITCH}$. Thus, in particular, if $n$ is large, the meta-slot scheme achieves a lower hardware cost.

\section{Conclusion}

In this paper, the meta-slot scheme, which was developed for nonblocking elastic optical networks, was investigated. The paper focused on the problems that were not addressed in the previous study [10]. The problems include the metaslot class size optimization for WSW1 as well as the class size optimization and assignment of meta-slots for WSW2. For WSW1, the objective is the number of FSUs required on an inner link for the network to be nonblocking. Meanwhile, for WSW2, the objective function is the number of S-switches required for nonblocking connections. By minimizing these objectives, it is possible to decrease the hardware costs of WSW1 and WSW2. 
For WSW1, the paper first proved that the meta-slot class size examined in [10] is not optimal. Next, it was elucidated that the class sizes are efficiently optimized by solving the shortest path problem in a network, which models the relationship between the class sizes and the number of FSUs.

The meta-slot scheme optimized by the shortest path algorithm was compared with the SNB condition [6] and the previously reported WSNB conditions [9]. The result indicates that the number of FSUs required on an inner link is smaller for the optimized meta-slot scheme than for the SNB and previously reported WSNB conditions, particularly if the number of FSUs on an outer link is large.

The objective for the WSW2 optimization depends on the number of FSUs required for an inner link and the assignment of the meta-slots to each S-switch. Among these factors, it was shown that the shortest path model minimizes the number of FSU on an inner link. Furthermore, it was also revealed that the optimal assignment of meta-slots among Sswitches is modeled as a bin-packing problem. Finally, the meta-slot scheme, designed by the shortest path algorithm and the approximate bin-packing algorithm, was compared with the previously reported SNB and WSNB conditions. The result reveals the advantage of the meta-slot scheme.

The meta-slot optimization presented in this paper employs the well-known problem models, such as the shortest path and bin-packing models. Since efficient algorithms are known for these problems, the presented method is quickly executable. In addition, the evaluation results indicate the superiority of the optimized meta-slot scheme over the previously reported WSNB conditions. Thus, it is concluded that the meta-slot scheme optimized by the proposed method is very promising for an efficient operation of WSW1 or WSW2 elastic optical switching networks.

\section{Compliance with ethical standards}

\section{Acknowledgments}

This work was supported by JSPS KAKENHI Grant Number JP19K11928. The author would like to thank Enago (www.enago.jp) for the English language review.

\section{Disclosure of conflict of interest}

The author declares no conflict of interest.

\section{References}

[1] Jinno M. Elastic optical networking: roles and benefitsinbeyond100-Gb/s era. Journal of Lightwave Technology. 2017; 35(5): 1116-1124.

[2] Chaterjee B, Sarma N, Oki E. Routing and spectrum allocation in elastic optical networks: a tutorial. IEEE Communication Surveys \& Tutorials. 2015; 17(3): 1776-1800.

[3] Jinno M, Kozicki B, Takara H, Watanabe A, Sone Y, Tanaka T, Hirano A. Distance-adaptive spectrum resource allocation in spectrum sliced elastic optical path network. IEEE Communications Magazine. 2010; 48(8): 138145.

[4] ITU-T. Spectral grids for WDM applications: DWDM frequency grid. ITU-T Recommendation G694. $2012 ; 1$.

[5] Kabaciński W, Michalski M, Abdulsahib M. The strict-sense nonblocking elastic optical switch. In: proc. HPSR2015, Budapest, Hungary. 2015.

[6] Kabaciński W, Michalski M, Rajewski R. Strict-sense nonblocking W-S-W node architecture for elastic optical networks. Journal of Lightwave Technology. 2016; 34(13): 3155-3162.

[7] Kabaciński W, Al-Tameemi A, Rajewski R. Rearrangeability of wavelength-space-wavelength switching fabric architecture for elastic optical switches. IEEE Access. 2019; 7: 64993-65006.

[8] Lin BC. Rearrangeable W-S-W elastic optical networks generated by graph approaches. Journal of Optical Communications and Networking. 2018; 10(8): 675-685.

[9] Kabaciński W, Abdulsahib M, Michalski M. Wide-sense nonblocking W-S-W node architectures for elastic optical networks. IEICE Transactions on Communications. 2019; E102-B(5): 978-991. 
[10] Ohta S. Meta-slot schemes to enhance nonblocking elastic optical switching networks. In: proc. the 2019 International Conference on Advanced Technologies for Communications (ATC 2019), Hanoi, Vietnam. 2019; 252-257.

[11] Danilewicz G, Kabaciński W, Rajewski R. Strict-sense nonblocking space-wavelength-space switching fabrics for elastic optical network nodes. Journal of Optical Communication Networks. 2016; 8(10): 745-756.

[12] Lin BC. Rearrangeable and repackable S-W-S elastic optical networks for connections with limited bandwidths. Applied Sciences. 2020; 10: 1-9.

[13] Kabaciński W, Michalski M, Rajewski R, Zal M. Optical datacenter net-works with elastic optical switch. In: proc. ICC2017, Paris, France. 2017.

[14] Stavdas A, Bianco A, Pattavina A, Raffaelli C, Matrakidis C, Piglione C, Politi C, Savi M, Zanzottera R. Performance evaluation of large capacity broadcast-and-select optical crossconnects. Optical Switching and Networking. 2011; 9: $13-24$.

[15] Jajszczyk A. Nonblocking, repackable, and rearrangeable Clos networks: fifty years of theory evolution. IEEE Communications Magazine. 2003; 41(10): 28-33.

[16] Hwang F. The mathematical theory of nonblocking switching networks, Second edition. Singapore: World Scientific; 2004.

[17] Coffman JEG, Garey M, Johnson D. Approximation algorithms for bin packing; a survey. In: Hochbaum DS eds. Approximation algorithms for NP-hard problems. Boston: PWS Publishing; 1997. 13-61.

[18] Dosa G, Li R, Han X, Tuza Z. Tight absolute bound for First-Fit Decreasing bin-packing: FFD(L) $\leq 11 / 9$ OPT(L) +6/9. Theoretical Computer Science. 2013; 510: 13-61.

[19] Marom DM, Colbourne PD, D’ Errico A, Fontaine NK, Ikuma Y, Proietti R, Zong L, Rivas-Moscoso JM, Tomkos I. Survey of photonic switching architectures and technologies in support of spatially and spectrally flexible optical networking. Journal of Optical Communications and Networkin. 2017; 9(1): 1-26.

[20] White I, Penty R, Webster M, Chai YJ, Wonfor A, Shahkooh S. Wavelength switching components for future photonic networks. IEEE Communications Magazine. 2002; 40(9): 74-81.

[21] Xie D, Wang D, Zhang M, Liu Z, You Q, Yang Q, Yu A. LCoS-based wavelength-selective switch for future finer-grid elastic optical networks capable of all-optical wavelength conversion, IEEE Photonics Journal. 2017; 9(2): 1-12.

[22] Clos C. A study of nonblocking switching networks. Bell System Technical Journal. 1953; 32(2): 406-424.

[23] Paull MC. Reswitching of connection networks. Bell System Technical Journal. 1962; 41(3): 833-855.

[24] Beneš VE. Mathematical theory of connecting networks and telephone traffic. New York: Academic Press; 1965.

[25] Tsai KH, Wang DW, Hwang F. Lower bounds for wide-sense non-blocking Clos network. Theoretical Computer Science. 2001; 261: 323-328.

[26] Chang F, Guo J, Hwang F, Lin C. Wide-sense nonblocking for symmetric or asymmetric 3-stage Clos networks, Theoretical Computer Science. 2004; 314: 375-386.

[27] Zhong W, Lacey J, Tucker R. Multiwavelength cross-connects for optical transport networks. Journal of Lightwave Technology. 1996; 14(7): 1613-1620.

[28] Koga M, Watanabe A, Kawai T, Sato K, Ohmori Y. Large-capacity optical path cross-connect system for WDM photonic transport network. IEEE Journal on Selected Areas in Communications. 1998; 16(7): 1260-1269.

[29] Konoike R, Suzuki K, Inoue T, Matsumoto T, Kurahashi T, Uetake A, Takabayashi K, Akiyama S, Sekiguchi S, Namiki S, Kawashima H, Ikeda K. SOA-integrated silicon photonics switch and its lossless multi-stage transmission of high-capacity WDM signals, Journal of Lightwave Technology. 2019; 37(1): 123-130.

[30] Siokis A, Christodoulopoulos K, Pleros N, Varvarigos E. Electro-optic switches based on space switching of multiplexed WDM signals: blocking vs non-blocking design trade-offs. Optical Switching and Networking. 2017; 25: 40-56.

[31] Turner J, Melen R. Multirate Clos networks, IEEE Communications Magazine. 2003; 41(10): 38-44.

[32] Ahuja RK, Magnanti TL, Orlin JB. Network flows, Upper Saddle River: Prentice-Hall; 1993. 